\title{
Análisis de la relación entre el temperamento y el aprendizaje léxico
}

Mayra Alejandra Villarreal Garza ${ }^{1}$

y Alberto Jorge Falcón Albarrán ${ }^{1}$

\section{Introducción}

La adquisición del lenguaje constituye una de las habilidades cognoscitivas más relevantes y complejas del ser humano. Gracias al dominio de una lengua, el hombre es capaz de crear y compartir contenidos del pensamiento, ya sea como emisor o receptor, logrando así la comunicación e integración del individuo a su grupo social y cultural. Esta habilidad es dividida en varios niveles, como lo son: el nivel pragmático, sintáctico, fonológico y léxico, siendo este último el objeto de nuestro estudio.

En las últimas décadas se ha estudiado el papel que juegan diferentes aspectos en el aprendizaje léxico (aprendizaje de nuevas palabras), uno de ellos es el temperamento, entendido como las características individuales que se asume que tienen una base genética que determinan las respuestas afectivas, atencionales y motoras del individuo en varias situaciones (Rothbarth, Kagan, Einsenberg, Calkins y Shiner, 2011). De esta definición se derivan 
tres dimensiones: extraversión, afecto negativo y control esforzado; las primeras dos se refieren a la reacción emocional del niño hacia el medio y la última se relaciona con aspectos cognitivos, como la regulación de la atención.

Investigaciones recientes demuestran que las dimensiones de control esforzado y extraversión predicen significativamente las variables del aprendizaje de nuevas palabras (Dixon y Shore, 1997; Dixon y Smith, 2000) y su comprensión (Morales et al., 2000). Otros estudios indican que los niños con temperamento difícil, incluyendo el bajo nivel de control esforzado y el alto efecto negativo, muestran ciertas desventajas en cuanto a lenguaje se refiere, en comparación con los niños con temperamento fácil (Salley y Dixon, 2007). Además, se menciona la dimensión de afecto negativo como indicador de atención conjunta, es decir, entre mayor afecto negativo, menor respuesta a ofertas de atención conjunta tendrá el niño.

Por otro lado, Todd y Dixon (2010) observaron algunos resultados paradójicos al analizar la dimensión de control esforzado, encontrando que los niños pobres en esta área tenían una mayor probabilidad de responder a ofertas de atención conjunta, lo cual resulta contradictorio con base en los resultados de investigaciones previas, ya que se afirmaba que los niños con altos niveles de control esforzado lograban responder de mejor manera a ofertas de atención conjunta y esto, a su vez, daba mayor nivel de adquisición de palabras.

Dentro de esta misma línea, encontramos el estudio que indica que los niños rápidos en habituarse visualmente son precisamente los niños con temperamento difícil, lo cual da como resultado mayor vocabulario (Dixon y Smith, 2000).

Por otra parte, Oakes, Kannass, y Shaddy (2002) evaluaron los efectos interactivos de factores endógenos (temperamento) y exógenos (características de los objetos) sobre la asignación de atención de los bebés mediante la evaluación de la familiaridad y la latencia hacia el distractor durante la exploración de objetos, encontrando que la asignación de la atención depende de las características de objetos, los objetivos del bebé, la motivación, el estado interno; además, encontraron 
mayores latencias hacia el distractor dependiendo del desarrollo del bebé.

En otro estudio, Dixon y Salley (2007) tenían como objetivo observar la relación entre el temperamento y el aprendizaje de palabras mediante una tarea de Fast Mapping. Para tales fines evaluaron a 47 niños de 22 meses de edad. La tarea consistía en presentar un objeto desconocido con cuatro ejemplares conocidos. Esta tarea era acompañada de dos distractores, uno social (persona leyendo un cuento detrás del infante) y uno mecánico (un juguete en forma de robot entrando al cubículo donde se llevaba a cabo la tarea). Al analizar los resultados, observaron que los niños con temperamento fácil lograban asignar la atención de manera eficiente y esto correlacionaba positivamente con el aprendizaje de una nueva palabra, es decir, niños con temperamento fácil mostraban ventajas respecto a niños con temperamento difícil en cuanto a aprendizaje de vocabulario se refiere, debido a los recursos atencionales usados en la tarea; sin embargo, estos autores afirman que los resultados obtenidos en su estudio pudieran ser debidos a la memoria de los sujetos, debido a que su método no permite medir de una manera rigurosa la atención que los infantes muestran tanto a los estímulos como a los distractores.

Por otro lado, Vlach y Sandhofer (2011) llevaron a cabo un experimento sobre una tarea de generalización de un sustantivo en donde el contexto de fondo era manipulado. Estos autores concluyen que a partir de los cuatro años, los niños demostraron alto rendimiento en todas las condiciones. Los resultados son discutidos en términos de la relación entre el aprendizaje de palabras y los procesos de memoria. Es decir, la adquisición de palabras en diversos contextos/distractores depende de la memoria del infante.

De acuerdo con lo anterior, se puede concluir que el temperamento tiene una influencia importante en el desarrollo lingüístico del ser humano; sin embargo, es un constructo que necesita mayor investigación, por lo cual es de suma importancia realizar investigaciones al respecto que ofrezcan mejores explicaciones sobre cómo diversos factores influyen en el desarrollo cognitivo del infante ( $y$ que por lo tanto permitan realizar intervenciones mucho más integrales). 
El presente trabajo tiene como objetivo explorar la relación entre temperamento (específicamente la dimensión control esforzado, relacionada con la atención), tiempos de atención visual en una condición experimental, aprendizaje de nuevas palabras y desarrollo lingüístico.

\section{Método}

\section{Participantes}

Con este propósito se evaluó a 60 niños de entre 18 y 24 meses de edad, con el criterio de que su única lengua hablada en casa fuera el español y sin diagnóstico o sospecha sobre algún problema auditivo, perinatal o neurológico.

\section{Material}

1. The Early Childhood Behavior Questionnaire (ECBQ), inventario de 18 escalas con 201 ítems, está diseñado para medir el temperamento de los niños entre las edades de 18 y 36 meses de edad. Evalúa las siguientes dimensiones de temperamento: extraversión (impulsividad, nivel de actividad, placer de alta intensidad, sociabilidad, anticipación positiva), afecto negativo (incomodidad, miedo, tristeza, timidez, facilidad para ser tranquilizado, activación motora y sensibilidad perceptual) y control esforzado (control inhibitorio, cambio atencional, disfrutar de ser abrazado, atención focalizada y placer de baja intensidad).

Los ítems son respondidos por los padres o cuidadores del niño y cada afirmación describe situaciones que vive el infante en su vida diaria, por ejemplo: ¿Con qué frecuencia durante la última semana el bebé jugó con objetos durante 5-10 minutos?

Las respuestas describirán las conductas que presenta el niño ante dichas situaciones. Existen seis posibles res- 
puestas que van desde "nunca sucede" hasta "siempre" (Rothbart, 2006).

2. Los Inventarios de Desarrollo Comunicativo MacArthur reflejan el proceso normal de adquisición temprana del lenguaje mediante un conjunto de manifestaciones diversas: gestos prelingüísticos, vocalizaciones prelingüísticas, vocabulario y gramática. Consta de dos inventarios diferentes: vocalizaciones, primeras palabras y gestos (8 a 15 meses). Vocalizaciones, palabras y gramática (16 a 30 meses). El último inventario fue utilizado en la presente investigación, el cual consta de dos medidas, palabras que el niño comprende y palabras que el niño comprende y dice. Estos inventarios están pensados para ser cumplimentados por los padres o cuidadores (Ornat et al., 2005).

3. El Paradigma Intermodal de Atención Preferencial, PIAP, es una adaptación realizada por Golinkoff et al. (1987) del paradigma de atención visual desarrollado por Fantz (como se cita en Falcón, 2012), quien empleó dicho método en estudios sobre las preferencias entre imágenes que mostraban infantes tan pequeños como de dos meses de edad (Falcón, 2012). El procedimiento se basa en la presentación al infante de dos estímulos visuales (actualmente, en algunos laboratorios más de dos) y un estímulo auditivo correspondiente sólo a una de las imágenes. El propósito de una prueba aplicada bajo este paradigma es observar si el infante es capaz de identificar la imagen correspondiente al estímulo auditivo presentado (Falcón, 2012).

\section{Procedimiento}

En primer lugar se administrará a los padres el inventario sobre temperamento Early Childhood Behavior Questionnaire (ECBQ) (Rothbart, 2006). Con los resultados anteriores se categorizará a los infantes en niños con temperamento difícil y fácil, para después llevarlos a una tarea de aprendizaje de palabras mediante el paradigma intermodal de atención preferencial PIAP (Golinkoff et al., 1987). La tarea tendrá dos condiciones, una en donde el 
fondo de la pantalla donde se enseñan las nuevas palabras será del mismo color (contexto uniforme) y otra donde ese mismo fondo cambiará de color cada vez que se presente una nueva imagen (contexto variable).

Además, se obtendrá información sobre el desarrollo lingüístico de los infantes a través de la aplicación de un reporte parental sobre las palabras que comprenden y producen los infantes (Inventario de Desarrollo Comunicativo MacArthur).

\section{Resultados}

Para determinar si el temperamento juega un papel importante en el desarrollo lingüístico y como primera etapa de la obtención de los resultados, se realizó una correlación entre los resultados obtenidos de los reportes parentales acerca de temperamento (ЕСBQ) y desarrollo lingüístico (MacArthur). Este análisis reveló una correlación estadísticamente significativa (.576) entre el desarrollo lingüístico (específicamente la comprensión) y la dimensión control esforzado del temperamento.

\section{Discusión}

Los resultados encontrados al analizar el desarrollo léxico y el temperamento coinciden con los resultados encontrados por Morales et al. (2000), quienes relacionan positivamente el temperamento únicamente con el vocabulario receptivo, es decir, la comprensión.

\section{Referencias}

Dixon, W.E. y Smith, P.H. (2008). Attentional Focus Moderates Habituation-Language Relationships: Slow Habituation May Be Good Thing. Infant and Child Development 17, 95-108.

Dixon, W. y Salley, B. (2007). "Shhh! We're Tryin'to Concentrate": Attention and Enviromental Distracters in Novel Word Learning. The Journal of Genetic Psychology 167 (4), 393-414. 
Dixon, W. y Shore, C. (1997). Temperamental Predictors of Linguistic Style During Multiword Acquisition. Infant Behavior and Development 20(1), 99-103.

Dixon, W. y Smith, H. (2000). Links Between Early Temperament and Language Acquisition, 417-440.

Falcón, A.J. (2012). Bases cognitivas para la adquisición del sistema morfológico del lenguaje. México, D.F.: UnAm.

Golinkoff, R.M., Hirsch-Pasek, K., Cauley, K.M. y Gordon, L. (1987). The Eyes Have It: Lexical And Syntactic Comprehension in a New Paradigm. Journal of Child Language 14(1), 23-45.

Morales, M., Mundy, P., Delgado, C., Yale, M., Neal, R. y Schwartz, H. (2000). Gaze Following, Temperament and Language Development in 6 Month Olds: A Replication and Extension. Infant Behavior \& Development 23, 231-236.

Oakes, L., Kannass, K. y Shaddy, J. (2002). Developmental Changes in Endogenous Control of Attention: The Role of Target Familiarity on Infants' Distraction Latency. Child Development, 1644-1655.

Ornat, S., Gallego, C., Gallo, P., Karousou, A. y Mariscal, S. (2005). Inventario de Desarrollo Comunicativo MacArthur. Manual de la adaptación española.

Rothbarth, M.K., Kagan, J., Einsenberg, N., Calkins, S. y Shiner, R.L. (2011). Enciclopedia sobre el Desarrollo de la Primera Infancia. Colombia.

Rothbart, M.K. (2006). Mary Rothbart's. Temperament Questionnaire. Obtenido de http://www.bowdoin.edu/ sputnam/ rothbart-temperament-questionnaires/instrument-descriptions/infant-behavior-questionnaire.html.

Salley, B.J. y Dixon, W. (2007). Temperamental and Joint Attentional Predictors of Language. NIH Public Access, 131-154.

Todd, J. y Dixon, W. (2010). Temperament Moderates Responsiveness to Joint Attention in 11 Month Old Infants. Elsevier, 297-308.

Vlach, H. y Sandhofer, C. (2011). Developmental Differences in Children's Context-Dependent Word Learning. Journal of Experimental Child Psychology, 108, 394-401. 
